\title{
Distortion of femtosecond laser pulses in lenses
}

\author{
Z. Bor \\ Max-Planck-Institut für biophysikalische Chemie, Abteilung Laserphysik, Postfach 2841, D-3400 Göttingen, Federal Republic of Germany
}

Received June 24, 1988; accepted November 6, 1988

\begin{abstract}
Large temporal front distortion of femtosecond pulses occurs in lenses having chromatic aberration. The effect is due to the difference between the phase and group velocities. Equations describing the pulse-front delay in singlet lenses, achromats, and compound lenses are presented. The pulse-front delay is several orders of magnitude larger than the broadening caused by group-velocity dispersion in the lens material. Delays occurring in Fresnel-type zone plates are also described.
\end{abstract}

The temporal shape of an ultrashort pulse may change drastically if it is propagating through a medium with dispersion. The first derivative of the refractive index with respect to the wavelength determines the difference between the phase and group velocities. The second derivative determines the pulse broadening due to group-velocity dispersion (GVD). The propagation effects occurring on refraction on plane surfaces (e.g., in prism systems) have been studied in detail and are used successfully for intracavity and extracavity pulse compression..$^{1-4}$ However, only recently has attention been paid to the propagation effects occurring in lenses and lens systems. ${ }^{5-8}$

In this Letter the temporal and spatial distortion of a pulse front is described on traversing a lens or lens system. The distortion is closely related to chromatic aberration of the lenses.

Owing to the difference between the phase and group velocities, the pulse front-which is defined as the surface coinciding with the peak of a pulse-is delayed with respect to the phase front. This delay can be calculated as $\mathbf{s}^{3,9,10}$

$$
\Delta T(r)=\frac{l}{c}\left(-\lambda \frac{\mathrm{d} n}{\mathrm{~d} \lambda}\right),
$$

where $l$ is the path length of light in the medium having a refractive index of $n(\lambda)$.

In the range of transparency for the optical materials of practical interest, the typical value of $-\lambda(d n / d \lambda)$ is approximately $2-20 \% .11$ (Higher values correspond to the shorter wavelength toward the spectral cutoff.) This means that the pulse front is spatially delayed with respect to the phase front by $2-20 \%$ of the distance traveled in the medium. For femtosecond pulses such large delays cannot be neglected.

Temporal delay between the phase and pulse fronts also occurs in a lens when an ultrashort pulse traverses it. For example, a lens made from fused silica having a diameter of $80 \mathrm{~mm}$ and a focal length of $f=150 \mathrm{~mm}$ has a center thickness of $D=10.5 \mathrm{~mm}$. The dispersion of silica at the wavelength of the $\mathrm{KrF}$ excimer laser $(249 \mathrm{~nm})$ is $\lambda(\mathrm{d} n / \mathrm{d} \lambda)=-0.1375 .7,12$ This means that for the axial ray the pulse front is delayed by 4.81 psec [see Eq. (1)], while for the marginal ray for which the distance traveled in silica is zero no delay is encountered. From the above numerical example it is obvious that the pulse front may be drastically distorted and that a detector located at the focal plane of a lens perceives a considerably longer pulse.

By using Fermat's principle and applying analytical ray tracing in the paraxial approximation, it can be shown that the delay between the phase and pulse fronts can be calculated as

$$
\Delta T(r)=\frac{r_{0}^{2}-r^{2}}{2 c f(n-1)}\left(-\lambda \frac{\mathrm{d} n}{\mathrm{~d} \lambda}\right),
$$

where $r$ and $r_{0}$ are the input paraxial radii of an arbitrary and the marginal ray, respectively (Fig. 1). The above effect is proportional to $\mathrm{d} n / \mathrm{d} \lambda$ and is referred to as the propagation time difference (PTD). In Eq. (2) the delay for the marginal ray (i.e., $r=r_{0}$ ) is assumed to be zero.

Behind the focus of the lens, the pulse front is changing its shape from convex to concave (Fig. 1). It can be shown that at a distance of

$$
L=\frac{f}{n-1}\left(-\lambda \frac{\mathrm{d} n}{\mathrm{~d} \lambda}\right)
$$

the pulse front is flat, while the phase front has a radius of curvature of $L$. This plane is a preferred plane and in certain experiments can be used to cir-

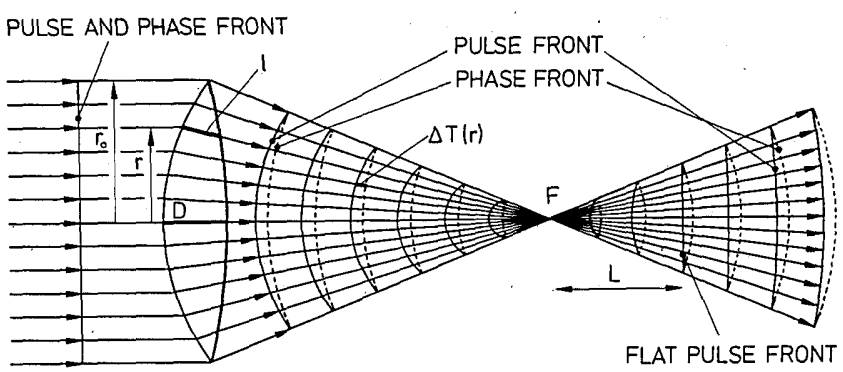

Fig. 1. Owing to the difference between the group and phase velocities, the pulse front is delayed with respect to the phase front. The delay is constant along one ray. At a distance $L$ behind the focus the pulse front is flat. 


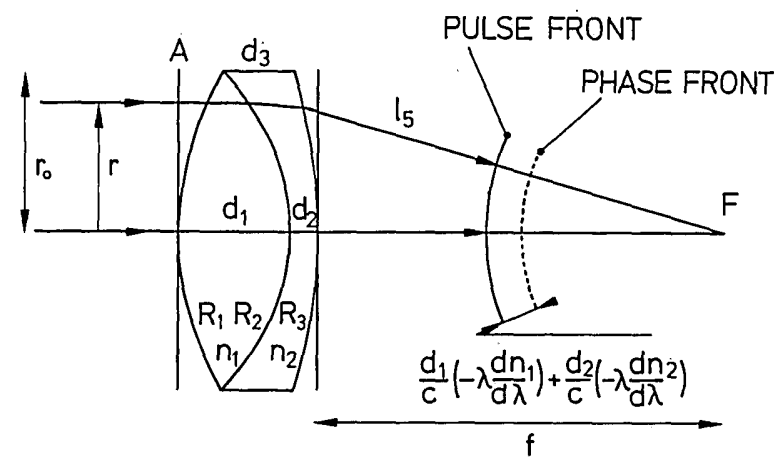

Fig. 2. For an achromat the delay between the pulse and phase fronts is constant over the cross section of the lens.

cumvent the unwanted effect of pulse-front distortion. For fused silica at $249 \mathrm{~nm}$, this plane is located at $L=$ $0.27 f$ behind the focus. (Note that the quantity $L / f$ depends only on the material of the lens.)

Calculations show ${ }^{7}$ that for an achromatic doublet the propagation time from plane $A$ to the focal point $F$ is

$$
T(r)=\frac{d_{1}}{c}\left(n_{1}-\lambda \frac{\mathrm{d} n_{1}}{\mathrm{~d} \lambda}\right)+\frac{d_{2}}{c}\left(n_{2}-\lambda \frac{\mathrm{d} n_{2}}{\mathrm{~d} \lambda}\right)+\frac{f}{c},
$$

where the relations among the symbols are shown in Fig. 2. In the derivation of Eq. (4), Fermat's principle and the condition of achromatism obtained from $\mathrm{d} f / \mathrm{d} \lambda$ $=0$ were used. 7 Thus, for an achromat, in contrast to singlet lenses, the propagation time is the same for any input radius $r$ [Eq. (4)]. By using the condition of achromatism, it can be shown ${ }^{7}$ that $T(r)$ can be expressed also as $T(r)=d_{3} / c\left(n_{2}-\lambda \mathrm{d} n_{2} / \mathrm{d} \lambda\right)+f / c$.

Next we calculate the pulse broadening due to GVD. For a singlet lens the broadening of the pulse due to GVD can be calculated as 7,10

$$
\Delta \tau(r)=\frac{\lambda}{c} \frac{\mathrm{d}^{2} n}{\mathrm{~d} \lambda^{2}} \Delta \lambda l(r),
$$

where $l(r)$ is the path length of a ray in the lens material (Fig. 1).

It is convenient to express the bandwidth of the pulse as $\Delta \lambda=\lambda /(2 N)$, where $N$ is the number of coherent optical cycles in the pulse. (For a transformlimited pulse, where the form factor does not differ greatly from unity, $N=c \tau / \lambda$.)

A detector or a target located at the focal plane perceives both the PTD [Eq. (2)] and pulse broadening due to GVD [Eq. (5)]. Therefore, it is instructive to express their ratio from Eqs. (1) and (5) as

$$
\frac{\Delta T(r)}{\Delta \tau(r)}=\frac{2}{A} N
$$

where $A$ is a quantity expressing the proportionality between the second and first derivative of the refractive index. ${ }^{11} A$ is slightly wavelength dependent and has a typical value between 2.6 and 4.0. (Higher values of $A$ correspond to a shorter wavelength toward the spectral cutoff. ${ }^{11}$ ) Using the polynomial given for the refractive index of fused silica, we calculate that at $249 \mathrm{~nm}, A=3.775 .{ }^{7,12}$ From Eq. (6) we calculate that for a 100 -fsec-long pulse for a fused-silica lens at 249 $\mathrm{nm}$ the exact value of the PTD is 64 times larger than broadening due to GVD.

For a singlet lens one can in general assume that for any input radius $r$ the effect of delay between the pulse and phase fronts is approximately $N$ times larger than the broadening of the pulse due to GVD of the lens material. The above approximation is correct with the precision of a factor of $2 / A$.

For an achromat the pulse broadening due to GVD observed in the focal plane can be calculated as

$$
\Delta \tau=\frac{\mathrm{d} T(r)}{\mathrm{d} \lambda} \Delta \lambda,
$$

where $T(r)$ is given by Eq. (4). Taking the derivative of Eq. (4) with respect to $\lambda$, we obtain

$$
\Delta \tau(r)=\frac{\lambda^{2}}{2 c N}\left(d_{1} \frac{\mathrm{d}^{2} n_{1}}{\mathrm{~d} \lambda^{2}}+d_{2} \frac{\mathrm{d}^{2} n_{2}}{\mathrm{~d} \lambda^{2}}\right) .
$$

Thus for an achromat the broadening of the pulse due to GVD is independent of the input radius $r$. Therefore, it can be compensated for by grating ${ }^{13}$ or prism compressors. ${ }^{1-4}$

In Ref. 7 a more general expression for the delay between the phase and pulse fronts has been derived:

$$
\Delta T(r)=\frac{r_{0}^{2}-r^{2}}{2 c f^{2}} \lambda \frac{\mathrm{d} f}{\mathrm{~d} \lambda},
$$

where $d f / d \lambda$ is the longitudinal chromatic aberration of the lens. Equation (9) may be useful for the calculation of the PTD because the longitudinal chromatic aberration is usually specified by the suppliers of lenses. In the paraxial approximation, Eq. (9) is valid for singlet lenses, achromatic doublets, not fully compensated achromats, and, as shown below, Fresneltype focusing zone plates.

A zone plate (Fig. 3) for which the radii of the zones are given by

$$
\rho_{i}=\rho_{1} \sqrt{i}
$$

has a first-order focal length ${ }^{14}$ of

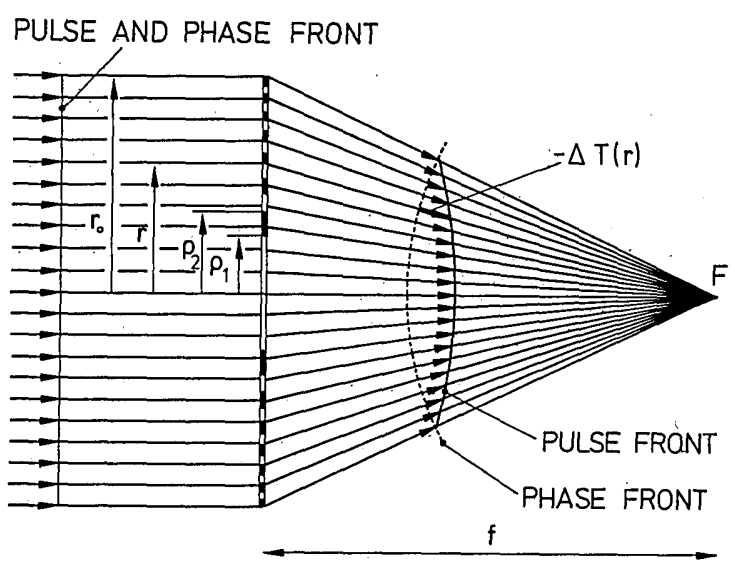

Fig. 3. For a focusing zone plate the pulse front is ahead of the phase front. The delay can be calculated using Eq. (9), obtained for refractive lenses. 


$$
f=\rho_{1}^{2} / \lambda
$$

In the paraxial approximation (i.e., $r_{0} / f \ll 1$; see Fig. 3 ) the delay between the pulse and phase fronts can be obtained after some trigonometry,

$$
\Delta T(r)=-\frac{r_{0}^{2}-r^{2}}{2 f c}
$$

The negative sign in Eq. (12) expresses that the pulse front is ahead of the phase front (Fig. 3) and, as in Eq. (2), the delay for the marginal ray is regarded to be zero.

Taking the derivative of Eq. (11) with respect to $\lambda$, we obtain the longitudinal chromatic aberration of the zone plate,

$$
\frac{\mathrm{d} f}{\mathrm{~d} \lambda}=-\frac{f}{\lambda}
$$

Combining Eqs. (12) and (13), we obtain the same expression as that obtained for a usual refractive lens [Eq. (9)].

It is surprising that Eq. (9) is also valid for a Fresneltype focusing zone plate in which the delay between the pulse and phase fronts has a different origin compared with that of a usual refractive lens. This is a hint that Eq. (9) has a more fundamental physical meaning. It can be shown ${ }^{15}$ that the general expression [Eq. (9)] is the manifestation of the uncertainty principle for a spectrograph consisting of a lens with chromatic aberration and a pinhole in the focus of the lens.

In conclusion, the most important properties of the pulse-front distortion occurring in lenses are summarized below:

(1) Owing to the difference between the phase and group velocities, the pulse front is delayed with respect to the phase front (Fig. 1). The delay is a parabolic function of the input beam radius [see Eq. (2)].

(2) For positive lenses, the pulse front is flat at a distance $L$ behind the focal plane given by Eq. (3). For negative lenses this plane is before the focal plane.

(3) For singlet lenses the pulse broadening caused by GVD is in practice several orders of magnitude smaller than the delay due to the difference between the phase and group velocities [see Eq. (6)].

(4) The general equation expressing the delay through the longitudinal chromatic aberration [Eq. (9)] is valid for a Fresnel-type focusing zone plate as well.

(5) Achromats have the valuable property that the delay between the pulse and phase fronts is constant over the lens cross section [see Eq. (4) and Fig. 2]. In most experiments, this is equivalent to no delay between the two fronts. Thus, the application of achromats for most experiments can eliminate the unwanted effects of pulse-front distortion.

(6) For achromats, the pulse broadening caused by GVD is larger than for singlet lenses. However, it is constant over the cross section of the lens [see Eq. (8)]. Such spatially homogeneous pulse broadening can be compensated for by pulse compressors.

The consideration of effects listed above are particularly important in designing large-aperture, high- $F$ number UV optics for inertial confinement fusion experiments and $x$-ray laser pumping.

The author is grateful to F. P. Schäfer for stimulating discussions and J. Jethwa for critical reading of the manuscript. This research has been supported by the German-Hungarian Exchange Program and the Országos Tudományos Kutatási Alap Foundation of the Hungarian Academy of Sciences.

The author's permanent address is Research Group on Laser Physics of the Hungarian Academy of Sciences, Dóm tér 9, H-6720 Szeged, Hungary.

\section{References}

1. O. E. Martinez, J. P. Gordon, and R. L. Fork, J. Opt. Soc. Am. A 1, 1003 (1984).

2. R. L. Fork, O. E. Martinez, and J. P. Gordon, Opt. Lett. 9, 159 (1984).

3. Z. Bor and B. Rácz, Opt. Commun. 54, 165 (1985).

4. J. D. Kafka and T. Baer, Opt. Lett. 6, 401 (1987).

5. J. Ihlemann, A. Helmbold, and H. Staerk, "Chromatic time lag in picosecond-streak-camera objectives," Rev. Sci. Instrum. (to be published).

6. H. Staerk, J. Ihlemann, and A. Helmbold, Laser Optoelektron. 20,6 (1988).

7. Z. Bor, "Distortion of femtosecond laser pulses in lenses and lens systems," J. Mod. Opt. (to be published).

8. S. Szatmári and G. Kühnle, "Pulse front and pulse duration distortion in refractive optics, and its compensation," Opt. Commun. (to be published).

9. M. R. Topp and G. C. Orner, Opt. Commun. 13, 276 (1975).

10. L. G. Cohen and C. Lin, Appl. Opt. 16, 3136 (1977).

11. Z. Bor and B. Rácz, Appl. Opt. 24, 3440 (1985).

12. I. H. Malitson, J. Opt. Soc. Am. 55, 1205 (1965).

13. E. B. Treacy, IEEE J. Quantum Electron. QE-5, 454 (1969).

14. R. W. Ditchburn, Light (Academic, New York, 1976).

15. Z. Bor, Am. J. Phys. (to be published). 